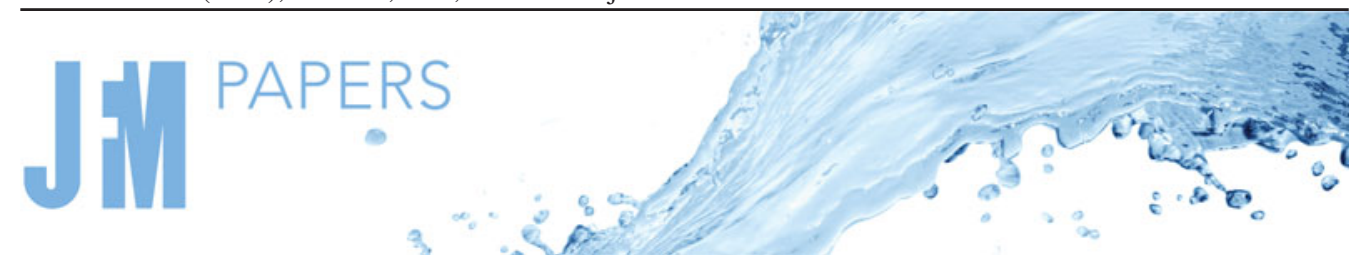

\title{
Rossby wave energy: a local Eulerian isotropic invariant
}

\author{
R.C. Kloosterziel ${ }^{1}$ and L.R.M. Maas $^{2, \dagger}$ \\ ${ }^{1}$ School of Ocean \& Earth Science \& Technology, University of Hawaii, Honolulu, HI 96822, USA \\ ${ }^{2}$ Institute for Marine \& Atmospheric Research, Utrecht University, Princetonplein 5, \\ 3584CC Utrecht, The Netherlands
}

(Received 2 February 2020; revised 1 December 2020; accepted 4 January 2021)

Conservation laws that relate the local time-rate-of-change of the spatial integral of a density function to the divergence of its flux through the boundaries of the integration domain provide integral constraints on the spatio-temporal development of a field. Here we show that a new type of conserved quantity exists that does not require integration over a particular domain but which holds locally at any point in the field. This is derived for the pseudo-energy density of non-divergent Rossby waves where local invariance is obtained for (i) a single plane wave, and (ii) waves produced by an impulsive point source of vorticity. The definition of pseudo-energy used here consists of a conventional kinetic part, as well as an unconventional pseudo-potential part, proposed by Buchwald (Proc. R. Soc. Lond. A, vol. 328, issue 1572, 1972, pp. 37-48). The anisotropic nature of the energy flux that appears in response to the point source further clarifies the role of the beta plane in the observed western intensification of ocean currents.

Key words: ocean circulation, waves in rotating fluids, vortex dynamics

\section{Introduction}

Conservation laws play a role in constraining the evolution of a partial differential equation $\mathcal{L} \psi=s$, say (Vanneste $\&$ Shepherd 1998). Here $\mathcal{L}$ defines a spatio-temporal differential operator and $s$ a given spatio-temporal source field. For a single dependent field variable $\psi(\boldsymbol{x}, t)$, varying in space $\boldsymbol{x}$ and time $t$, conservation laws take the form

$$
\frac{\partial E(\psi)}{\partial t}+\nabla \cdot \boldsymbol{F}(\psi)=0
$$

$†$ Email address for correspondence: 1.r.m.maas@uu.nl

(C) The Author(s), 2021. Published by Cambridge University Press. This is an Open Access article, distributed under the terms of the Creative Commons Attribution licence (http://creativecommons.org/ licenses/by/4.0/), which permits unrestricted re-use, distribution, and reproduction in any medium, provided the original work is properly cited. 


\section{R.C. Kloosterziel and L.R.M. Maas}

where $E(\psi)$ represents a density function and $F(\psi)$ a corresponding flux that depend on $\psi$ and its derivatives. These conservation laws imply the existence of an invariant, $\bar{E} \equiv \int_{D} E \mathrm{~d} x$. This invariant is obtained by integrating the conservation law over a fixed spatial domain $D$. Using Gauss' law, the domain integral of the divergence of the flux can be written as a boundary integral $\oint_{\partial D} \boldsymbol{F} \cdot \mathrm{d} \boldsymbol{n}$ of the flux's normal component across the boundary $\partial D$. Provided there is no net flux across this boundary, the domain-integrated density is time-invariant. To contrast this type of invariant with another type, obtained in the subsequent part of this paper, we will refer to this as a global Eulerian invariant. Note that this global invariant is only loosely related to space. It pertains to the domain over which the integration is performed, chosen such that there is no net flux through the boundary.

In this paper, we show that for the barotropic, non-divergent Rossby wave equation solutions exist for which the time derivative of the wave's total pseudo-energy, $E$, vanishes identically. This happens at any location in the field, $\partial E(\psi(\boldsymbol{x}, t)) / \partial t=0$, so that spatial integration and an appeal to Gauss' law become unnecessary. In other words, despite the fact that the underlying field $\psi(\boldsymbol{x}, t)$ varies in space and time, $E(\psi(\boldsymbol{x}, t))$ becomes a local Eulerian invariant. Obviously, as local invariance implies global invariance, the presence of a local Eulerian invariant provides a stronger constraint than its global counterpart. For a local Eulerian invariant, the conservation law reduces to a continuity equation, $\boldsymbol{\nabla} \cdot \boldsymbol{F}(\psi)=0$, implying that the flux vector $\boldsymbol{F}$ is incompressible (solenoidal). The first example we discuss is the trivial monochromatic plane Rossby wave. The second example, however, is the complicated Rossby wave response to an impulsive (instantaneous), infinitely concentrated forcing.

Non-divergent Rossby waves, the simplest type of waves carried by a non-uniformly rotating fluid, considered here, are usually described as possessing only kinetic energy. The potential energy of a Rossby wave is commonly related to work performed by or against gravity. This results from vertical displacements of a non-rigid surface, in which case the Rossby waves are divergent. Buchwald (1972), however, proposed that some form of Rossby wave potential energy (spin energy) may still be defined, even for non-divergent waves (Thomson 1973; LeBlond \& Mysak 1978). Slightly rewriting Buchwald's definition, this spin energy can be written as a quadratic functional of the streamfuntion with which non-divergent Rossby waves are described. Owing to its unusual character as potential energy and an ongoing debate whether it may qualify as a physical form of potential energy, as well as to its appearance in the Lagrangian in a variational derivation of the non-divergent Rossby wave equation $(\$ 2)$, we here refer to this as the wave's pseudo-potential energy.

In $\S 2$, we discuss conservation laws for free non-divergent Rossby waves and show that for a plane monochromatic non-divergent Rossby wave, the sum of kinetic $T$ and pseudo-potential energy $V$, the pseudo-energy $E=T+V$, is conserved at any location and in any phase of the wave, i.e. $\partial E / \partial t=0$. Section 3 discusses the non-trivial response - the Green's function - to an impulsive point source, as well as its near- and far-field limits. The Green's function shows that the response evolves from initially circular into a well-known series of 'banana'-shaped structures that have their opening towards the west (Veronis 1958; Longuet-Higgins 1965; Dickinson 1978) and that continuously reduce their scale, see figure 1. A detailed description and derivation of this exact solution to the forced Rossby wave equation is in Kloosterziel \& Maas (2017). In §4, the kinetic and pseudo-potential energy distributions of the Green's function are shown to also complement each other at any moment in time, although the initial response is entirely kinetic in the form of a circular potential flow. As for the plane monochromatic wave, we find that the spatial distribution of the total pseudo-energy $E$ is invariant in time 
and isotropic in space, showing that energy is locally conserved yet displaying a flux of energy towards infinity. Section 5 discusses the outward-directed, anisotropic energy flux due to the impulsive point source. We summarize our findings in $\S 6$, discussing also its implication for western intensification of ocean currents.

\section{Linear Rossby wave theory}

The simplest description of a planetary Rossby wave on a beta plane for a rigid-lid, homogeneous-density ocean or atmosphere is in linear approximation given by

$$
\partial_{t} q+\beta v=\operatorname{curl} \boldsymbol{\tau}
$$

Throughout this paper we set density $\rho=1$ for convenience. Here, relative vorticity $q=$ $\partial_{x} v-\partial_{y} u$ is created by south-north advection of planetary vorticity and by $\tau=\left\{\tau_{x}, \tau_{y}\right\}$ the wind-stress vector, see e.g. Veronis (1958) and Pedlosky (1987). As usual, $t$ is time, $x$ longitude, $y$ latitude and $\beta v$ is the advection of planetary vorticity by the latitudinal (south-north) velocity component $v$, where $\beta$ represents the magnitude of the northward directed planetary vorticity gradient.

Incompressibility, $\boldsymbol{\nabla} \cdot \boldsymbol{u}=0$, allows introducing a streamfunction $\psi$, so that the velocity components are $u=-\partial_{y} \psi, v=\partial_{x} \psi$ and $q=\nabla^{2} \psi$, with $\nabla^{2}=\left(\partial_{x}^{2}+\partial_{y}^{2}\right)$. 'Free' Rossby waves are governed by (2.1) with $\operatorname{curl} \tau=0$ on the right-hand side:

$$
\partial_{t} \nabla^{2} \psi+\beta \partial_{x} \psi=0
$$

Multiplication of (2.2) by $\psi$ yields a conservation law for kinetic energy

$$
T=\frac{1}{2} \nabla \psi \cdot \nabla \psi
$$

that reads

$$
\frac{\partial T}{\partial t}+\nabla \cdot \boldsymbol{F}_{T}=0 \quad \text { with } \boldsymbol{F}_{T}=-\psi \frac{\partial \boldsymbol{\nabla} \psi}{\partial t}-\boldsymbol{i} \frac{1}{2} \beta \psi^{2},
$$

where we use $i, j$ to denote the customary unit vectors associated with Cartesian $x, y$-directions, respectively. Buchwald (1972) established by inspection of (2.2) that the Rossby wave equation gives rise to a second conservation law, related to

$$
V=\frac{1}{2} \beta \psi \eta
$$

which acts as the density function of pseudo-potential energy per unit mass, despite the presence of a rigid upper surface. Here, $\eta$ denotes the northward particle displacement whose time derivative yields the northward velocity $v=\partial \eta / \partial t$.

Without use of Buchwald's results, the conservation equation for pseudo-potential energy $V$ can be obtained also by using the observation by Seliger \& Whitham (1968) that the linear non-divergent Rossby wave equation (2.2) follows with a variational principle akin to Hamilton's principle in classical mechanics: the dynamics is determined by requiring the difference of kinetic energy and potential energy to be stationary with respect to small variations.

By introducing an auxiliary function $\chi$, such that $\psi=\partial_{t} \chi$, Seliger \& Whitham (1968) note that (2.2) is obtained by demanding that the variation $\delta \mathrm{I}$ of an action integral $\mathrm{I}=$ $\iiint L \mathrm{~d} x \mathrm{~d} y \mathrm{~d} t$ with respect to small variations $\delta \chi$ vanishes. Here, $L$ is the Lagrangian 
density

$$
L=T-V=\frac{1}{2}\left(\frac{\partial \nabla \chi}{\partial t}\right)^{2}-\frac{1}{2} \beta \frac{\partial \chi}{\partial t} \frac{\partial \chi}{\partial x} .
$$

With the definition of $\chi$ we have $\psi=\partial_{t} \chi$ but also $\partial_{x} \chi=\eta$ because northward velocity $v=\partial_{t} \eta=\partial_{x} \psi=\partial_{x, t}^{2} \chi$. Thus $V$ in (2.6) equals the pseudo-potential energy (2.5) of Buchwald (1972). As Olbers, Willebrand \& Eden (2012) point out, a pseudo-potential energy appearing in the expression for the Lagrangian density is not necessarily identical to a physical energy, although the interpretation of (pseudo) kinetic energy $T$ as a physical energy is beyond dispute.

Using the Rossby wave equation (2.2), $V$ in (2.5) can be written elegantly as

$$
V=-\frac{1}{2} \psi \nabla^{2} \psi=-\frac{1}{2} \psi q \quad \text { with } q=\nabla^{2} \psi
$$

Definitions (2.3) and (2.7) show that the Lagrangian density can be written as the divergence of a flux:

$$
L \equiv T-V=\frac{1}{2} \nabla \cdot(\psi \nabla \psi)
$$

Subtracting the time derivative of $T-V$ in (2.8) from (2.4) yields the conservation law for pseudo-potential energy $V$ :

$$
\frac{\partial V}{\partial t}+\nabla \cdot \boldsymbol{F}_{V}=0 \quad \text { with } \boldsymbol{F}_{V}=\frac{1}{2} \frac{\partial \psi}{\partial t} \nabla \psi-\frac{1}{2} \psi \nabla \frac{\partial \psi}{\partial t}-i \frac{1}{2} \beta \psi^{2} .
$$

Since $E=T+V$, combining (2.4) and (2.9) implies the energy conservation law

$$
\frac{\partial E}{\partial t}+\nabla \cdot \boldsymbol{F}=0 \quad \text { with } \boldsymbol{F}=\boldsymbol{F}_{T}+\boldsymbol{F}_{V}=-\frac{3}{2} \psi \frac{\partial \boldsymbol{\nabla} \psi}{\partial t}+\frac{1}{2} \frac{\partial \psi}{\partial t} \nabla \psi-\boldsymbol{i} \beta \psi^{2} .
$$

The relevance of acknowledging $V$ as a form of pseudo-potential energy in (2.7) is immediately clear if we apply it to a free monochromatic Rossby wave: $\psi=A \sin \Phi$ with amplitude $A$ and phase $\Phi \equiv k x+l y-\omega t-\phi$, where wave vector $\boldsymbol{k}=(k, l)$ obeys the dispersion relation

$$
\omega=-\beta \frac{k}{\kappa^{2}}, \quad \kappa=|k|, \quad \omega>0 .
$$

Both kinetic and pseudo-potential energy,

$$
T=\frac{1}{2} \kappa^{2} A^{2} \cos ^{2} \Phi \quad \text { and } \quad V=\frac{1}{2} \kappa^{2} A^{2} \sin ^{2} \Phi
$$

propagate on average with the group velocity $\boldsymbol{c}_{g}=(\partial \omega / \partial k, \partial \omega / \partial l)$. These expressions do not only imply, as Buchwald inferred, equipartitioning of their phase-averages, where the phase-average is defined as $\langle\cdot\rangle=(1 / 2 \pi) \int_{0}^{2 \pi} \cdot d \Phi$, but they also render the total pseudo-energy

$$
E=T+V=\frac{1}{2} \kappa^{2} A^{2}
$$

a spatially uniform constant, without the need for any phase-averaging. Remarkably, an infinite-dimensional system thus seems to recover a local balance between kinetic and pseudo-potential energy, a property otherwise restricted to single degree of freedom systems, such as the pendulum. 
Rossby wave energy: a local Eulerian isotropic invariant

For plane non-divergent Rossby waves, the group velocity vector is given by

$$
c_{g}=\left(\frac{\partial \omega}{\partial k}, \frac{\partial \omega}{\partial l}\right)=i \frac{\beta\left(k^{2}-l^{2}\right)}{\kappa^{4}}+j \frac{2 \beta k l}{\kappa^{4}} .
$$

The energy flux vector

$$
\begin{aligned}
\boldsymbol{F} & =\frac{\beta A^{2}}{2 \kappa^{2}}\left(i\left[k^{2}-l^{2}+l^{2} \cos (2 \Phi)\right]+j l k[2-\cos (2 \Phi)]\right) \\
& =c_{g} E+\frac{1}{2} \beta A^{2} \frac{l}{\kappa^{2}} \cos (2 \Phi) \boldsymbol{k}^{\perp}
\end{aligned}
$$

where $\boldsymbol{k}^{\perp}=(l,-k)$ is a vector perpendicular to $\boldsymbol{k}$. This shows that, as usual in wave dynamics, the phase-average of the flux vector

$$
\langle F\rangle=c_{g} E
$$

is indeed the product of group velocity (2.14) and energy density (2.13). This spatially constant vector is obviously non-divergent. Yet it shows the presence of a steady flux of energy into the direction into which the group velocity points. The second, oscillatory part of the flux vector proportional to $\cos (2 \Phi)$, that accommodates the exchange of kinetic to pseudo-potential energy and back, consists of a vector that is perpendicular to wave vector $\boldsymbol{k}$, implying this part is indeed also non-divergent. Vanishing of the divergence of energy flux $\boldsymbol{F}$ in (2.15) agrees with the fact that pseudo-energy density $E$ is invariant over time. In $\S 4$, we show that this also holds for the much more involved response to an impulsive point source of vorticity which we shall first describe briefly in the next section.

\section{Rossby waves due to an impulsive point source: Green's function}

With $(2.12 a, b)$ we saw that for a plane monochromatic Rossby wave, the kinetic energy $T$ and pseudo-potential energy $V$ complement each other perfectly: where $T$ is high, $V$ is low and vice versa, in such a manner that their total sum $E$ is spatially uniform. In $\S 4$, we show that this also holds for the wave field generated by an instantaneous application of wind-stress torque, curl $\boldsymbol{\tau}$, infinitely concentrated at a singular point. This complicated Rossby wave field generated by an impulsive point source, the Green's function $G$, is described in detail in Kloosterziel \& Maas (2017). This Green's function is the solution of

$$
\mathcal{L} G=\delta(t) \delta(x) \delta(y) \quad \text { with } \mathcal{L}=\frac{\partial}{\partial t} \nabla^{2}+\beta \frac{\partial}{\partial x} .
$$

Here $\mathcal{L}$ is the Rossby wave operator and $\delta$ the usual Dirac delta-function. In Kloosterziel $\&$ Maas (2017), we showed that

$$
G=\frac{H(t)}{4}\left[\mathrm{~J}_{0}\left(z_{+}\right) \mathrm{Y}_{0}\left(z_{-}\right)+\mathrm{J}_{0}\left(z_{-}\right) \mathrm{Y}_{0}\left(z_{+}\right)\right],
$$

where $\mathrm{J}_{n}, \mathrm{Y}_{n}$ are $n$ th-order Bessel functions of the first and second kind, respectively, with complex conjugate arguments

$$
z_{ \pm}=\sqrt{\beta t(x \pm \mathrm{i} y)}=\sqrt{\beta \operatorname{tr}} \mathrm{e}^{ \pm \mathrm{i} \theta / 2},
$$

with $r, \theta$ the usual cylindrical coordinates in the $x, y$ plane. The complex conjugate variables $z_{ \pm}$can be expressed also in parabolic coordinates $\{\zeta, \eta\}$

$$
z_{+}=a z_{\star}, \quad z_{-}=a \bar{z}_{\star}, \quad a=\sqrt{\frac{\beta t}{2}}, \quad z_{\star} \equiv \zeta+\mathrm{i} \eta, \quad \bar{z}_{\star} \equiv \zeta-\mathrm{i} \eta
$$


with

$$
\zeta=\sqrt{x+r}, \quad \eta=\sqrt{r-x} \quad \text { with } r=\sqrt{x^{2}+y^{2}}, \quad \zeta^{2}+\eta^{2}=2 r .
$$

The Green's function has a self-similar dependence on $\beta$ tr, and an independent angular dependence on $\theta$. The prefactor $H(t)$ in (3.2) is the Heaviside unit stepfunction which enforces 'causality', i.e. $G=0$ for $t<0$. By definition $\delta(t) \equiv \mathrm{d} H(t) / \mathrm{d} t$. In the following, $H(t)$ will be omitted with the understanding that all results are for $t>0$. The Green's function is remarkable in that its closed-form expression requires the use of products of Bessel functions of complex arguments, even though the solution itself is real. Graphing $G$ given in (3.2) at various times is easy with available numerical packages, such as Wolfram Research, Inc. (2020).

Figure 1 displays the Green's function during its development. Initially, it consists of an isotropic response as the highest derivative term in the differential operator $\mathcal{L}$ in (3.1), representing the evolution of relative vorticity, which should match the point-source singularity. Hence, as on a non-rotating plane $(\beta=0)$, the fluid initially responds in the form of a circular irrotational point or line vortex. In figure $1(a)$, the near circular symmetry near the origin at the start is obvious. Mathematically, this initial behaviour and the behaviour near the origin follows with the small- $z$ behaviour of $J_{0}, Y_{0}$ in (3.2) (see (A1) in appendix A):

$$
\lim t r \downarrow 0: \quad G \sim \frac{1}{2 \pi} \ln r \quad \text { and swirl velocity } \quad v_{\theta}=\frac{\partial G}{\partial r} \sim \frac{1}{2 \pi r} .
$$

The point vortex's localized vorticity is positive, in agreement with the cyclonic nature of the applied curl of the wind stress. Outside the origin, the response initially consists of potential flow, lacking vorticity. Thus, initially all energy is kinetic, i.e. $V=0$, and with (3.6)

$$
\lim t \downarrow 0: E=T=\frac{1}{2} v_{\theta}^{2}=\frac{1}{2}\left(\frac{1}{2 \pi r}\right)^{2} .
$$

However, soon after, relative vorticity $q$ is created by cyclonic (counter-clockwise) advection of northwards-increasing background vorticity $(\beta v$ in $(2.1)$ or the second term of operator $\mathcal{L}$ in (3.1)). The exact expression for the vorticity $q=\nabla^{2} G$ is given below in $(4.3 a, b)$. In figure 2, we show graphs of $\nabla^{2} G$ at the same times for which $G$ is shown in figure 1 . Due to this advection, the secondary vortices acquire positive sign to the west, and negative sign to the east of the source. This is seen in the first two panels of figure 2 . At the central latitude $y=0$, these secondary vorticity fields in turn advect background vorticity, in both cases leading to positive tertiary vortices further to the west and to the east of the secondary vortices, respectively (see figure $2 c$ ). This process continues, to the east leading to an array of vortices alternating in sign, while those to the west are all positive. This leads to a single-signed, algebraically decreasing tail of vorticity to the west (proportional to $r^{-3 / 2}$ ) while to the east a set of westward propagating vortices appear.

The patterns seen in figure $1(c)$ for $G$ and figure $2(c)$ for $q=\nabla^{2} G$ at time $t=0.5$ can be described accurately by simple analytical expressions found with expansions of the Bessel function for large argument $z$ (see (A3) in appendix A). For example, for large $\beta$ tr

$$
G=\frac{1}{4}\left[\mathrm{~J}_{0}\left(z_{+}\right) \mathrm{Y}_{0}\left(z_{-}\right)+\mathrm{J}_{0}\left(z_{-}\right) \mathrm{Y}_{0}\left(z_{+}\right)\right] \sim-\frac{\cos \left(z_{+}+z_{-}\right)}{2 \pi \sqrt{z_{+} z_{-}}}=-\frac{\cos (\sqrt{2 \beta t(x+r)})}{2 \pi \sqrt{\beta t r}} .
$$

Lines of constant $\zeta=\sqrt{x+r}$ are parabolas (see figure 1) that open towards the west $(x<0)$. A number of these parabolas have been plotted in figure 1. Veronis (1958) already 
Rossby wave energy: a local Eulerian isotropic invariant
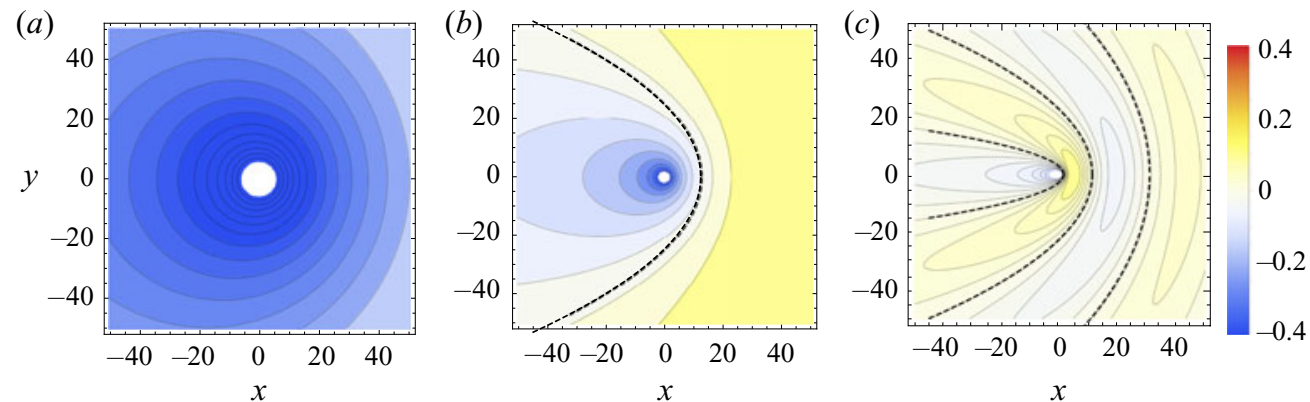

Figure 1. Green's function $G$ (3.2) for $(a) t=0.005$, (b) $t=0.05$ and (c) $t=0.5$ over a fixed spatial area, showing a decrease in magnitude and scale. The innermost dashed parabola in the second and third panels is defined by $2 a \zeta=(n+1 / 2) \pi$ with $n=0$ and $\zeta=\sqrt{x+r}, a=\sqrt{\beta t / 2}$. The neighbouring parabolas in the third panel are for $n=1$ and 2 , respectively.
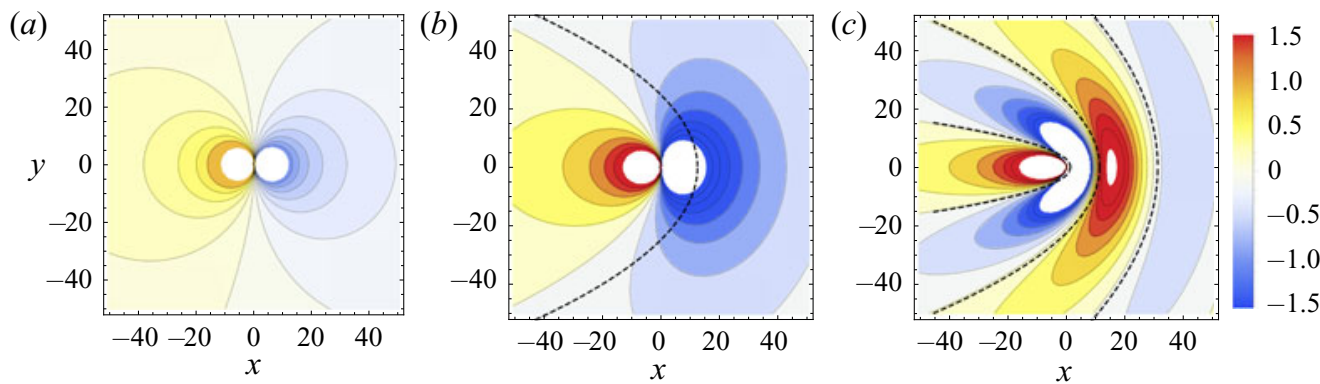

Figure 2. Vorticity of the Green's function, $q=\nabla^{2} G(4.3 a, b)$ at several moments in time $t$ over a fixed spatial area. Vorticity increases with time. To enable use of the same colour scale, $q$ is scaled by $\sqrt{t}$ and has been multiplied by $10^{3}$. Parabolas and times as in figure 1: (a) $t=0.005$, (b) $t=0.05$ and $(c) t=0.5$.

predicted that lines of constant phase would be such parabolas. For a more detailed description of $G$, see Kloosterziel \& Maas (2017).

With the advection of planetary vorticity a transfer of energy from $T$ to $V$ is enabled by means of Rossby waves, making it likely that also the total pseudo-energy, $E=T+V$, evolves. In the next section we show however that $E$ remains invariant as well as isotropic, i.e. at all times $E$ equals the initial kinetic energy (3.7).

\section{Energy for the Green's function wave field}

In the following, it will be expedient to use the following notation:

$$
\mathrm{I}_{m n} \equiv \mathrm{J}_{m}\left(z_{+}\right) \mathrm{Y}_{n}\left(z_{-}\right)+\mathrm{J}_{n}\left(z_{-}\right) \mathrm{Y}_{m}\left(z_{+}\right),
$$

where $m, n$ are integers. For brevity, we suppress the spatio-temporal dependence on the complex $z_{ \pm}$coordinates defined in (3.3). With this notation the Green's function (3.2) is

$$
G=\frac{1}{4} \mathrm{I}_{00} \text {. }
$$

Treating $G$ as the streamfunction $\psi$ of the forced problem $\mathcal{L} \psi=\delta(t) \delta(x) \delta(y)$, using $(3.4 a-e)$ it is easy to calculate the vorticity $q \equiv \nabla^{2} \psi=\nabla^{2} G$ :

$$
\nabla^{2}=\frac{4}{z_{\star} \bar{z}_{\star}} \frac{\partial^{2}}{\partial z_{\star} \partial \bar{z}_{\star}} \quad \text { and } \quad q=\nabla^{2} G=\frac{\beta t}{2\left(\zeta^{2}+\eta^{2}\right)} \mathrm{I}_{11}
$$


(a)

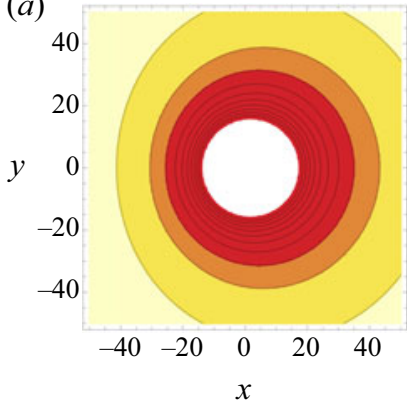

(b)

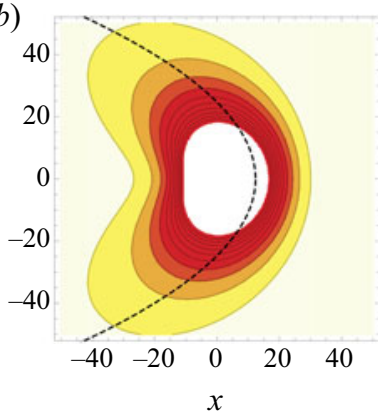

(c)

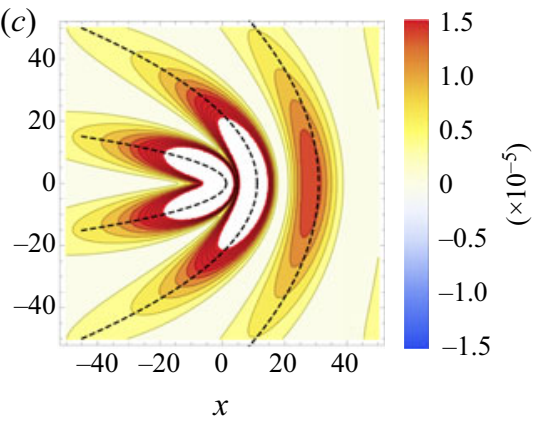

Figure 3. Kinetic energy of the Green's function, $T=(\nabla G \cdot \nabla G) / 2$ (4.4). Times as in previous figures 1 and 2: (a) $t=0.005$, (b) $t=0.05$ and $(c) t=0.5$. Parabolas also as in figures 1 and 2 .

(a)

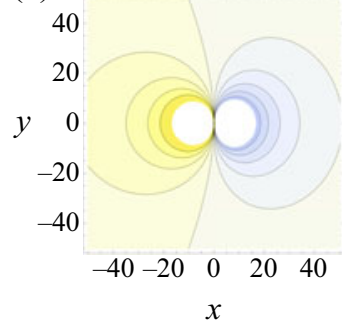

(b)

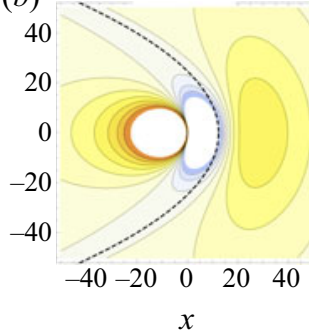

(c)

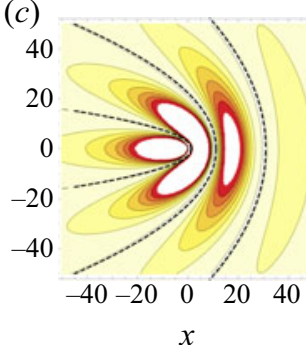

(d)

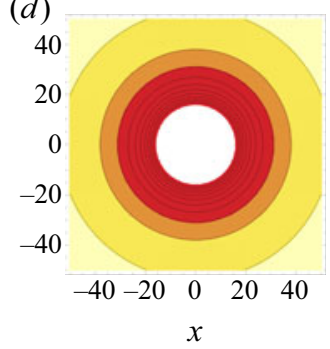

Figure 4. Pseudo-potential energy of the Green's function $V=-\left(G \nabla^{2} G\right) / 2(4.5)$ at times $(a) t=0.005$, (b) $t=0.05$ and $(c) t=0.5$ over a fixed spatial area. Parabolas and times as in figures 1,2 and 3 . Colour scale as in figure 3. Final panel $(d)$ shows the total energy $E=T+V$ (4.8), which is time-invariant and isotropic (see text).

because $\left\{\mathrm{J}_{0}^{\prime}, \mathrm{Y}_{0}^{\prime}\right\}=-\left\{\mathrm{J}_{1}, \mathrm{Y}_{1}\right\}$ (primes indicate differentiation). The variables $z_{\star}, \bar{z}_{\star}, \zeta, \eta$ are defined in (3.4a-e) and (3.5). Figure 2 shows this vorticity field at three instances. In Kloosterziel \& Maas (2017), we already determined that the kinetic energy $T=(\nabla \psi$ • $\nabla \psi) / 2$ associated with the streamfunction $\psi=G$ equals

$$
T=\frac{1}{2\left(\zeta^{2}+\eta^{2}\right)}\left[\left(\frac{\partial G}{\partial \zeta}\right)^{2}+\left(\frac{\partial G}{\partial \eta}\right)^{2}\right]=\frac{1}{4^{2}} \frac{\beta t}{\zeta^{2}+\eta^{2}} \mathrm{I}_{01} \mathrm{I}_{10}
$$

The evolution of the kinetic energy distribution $T$ is shown in figure 3. Late in its development, the kinetic energy's spatial distribution shows regions of very small values, see light yellow areas in figure $3(c)$. This suggests these must be regions where energy is represented by an appropriately defined form of potential energy. Substituting $\psi=G$ from (3.2) and $\nabla^{2} \psi=\nabla^{2} G$ from $(4.3 a, b)$ in (2.7) we find

$$
V=-\frac{1}{2} \psi \nabla^{2} \psi=-\frac{1}{2} G \nabla^{2} G=-\frac{1}{4^{2}} \frac{\beta t}{\zeta^{2}+\eta^{2}} \mathrm{I}_{00} \mathrm{I}_{11} .
$$

The pseudo-potential energy $V$ is shown in figure 4 for the same three times used in figure 3 for the kinetic energy $T$. Comparison of figure 4(c) with figure 3(c) shows that $V$ indeed complements $T$ in that where $T$ has low values, $V$ has high values and vice versa.

For reference, the same parabolas as in figure 1 have been shown as dashed lines in figures $3(b, c)$ and $4(b, c)$, passing through the maxima of $T$ and the minima of $V$. Note that the pseudo-potential energy can become negative, initially mimicking the vorticity's 
Rossby wave energy: a local Eulerian isotropic invariant
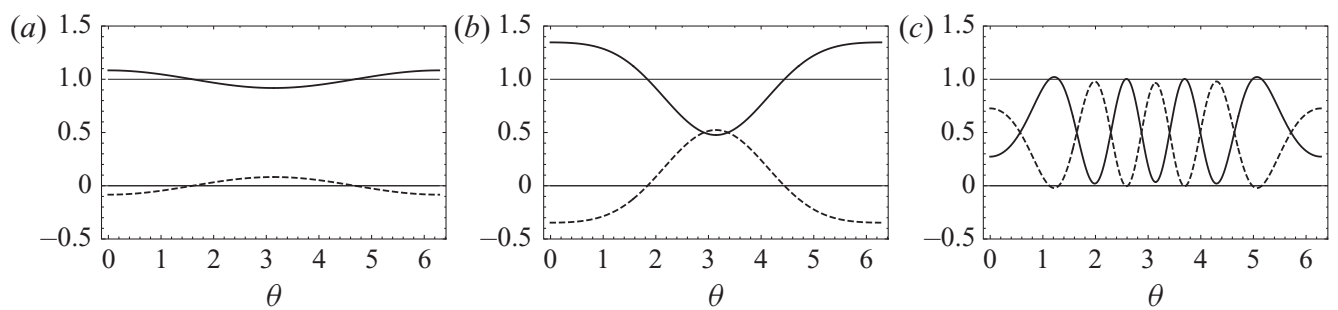

Figure 5. Angular distribution of normalized kinetic energy $T / E$ (solid) and pseudo-potential energy $V / E$ (dashed), scaled by total energy $E$ along circle of radius $r=40$ at times (a) $t=0.0005$, (b) $t=0.01$ and (c) $t=0.2$ (time increasing by a factor 20). While all energy $E$ starts out kinetic energy $T$, it rapidly converts into potential energy $V$ along the west axis $(\theta=\pi$, centre of graphs), but at all times $T / E+V / E=1$ (thin solid line).

sign (compare first panels of figures 2 and 4) because initially $-G>0$ everywhere, while, later, weak negative values of $V$ contract to regions where $T$ is maximal.

When (4.4) and (4.5) are added, four terms involving the product of four Bessel functions cancel and the result is

$$
\begin{aligned}
E= & T+V=\frac{1}{4^{2}} \frac{\beta t}{\zeta^{2}+\eta^{2}} \\
& \times\left[\mathrm{J}_{0}\left(z_{+}\right) \mathrm{Y}_{1}\left(z_{+}\right)-\mathrm{J}_{1}\left(z_{+}\right) \mathrm{Y}_{0}\left(z_{+}\right)\right]\left[\mathrm{J}_{0}\left(z_{-}\right) \mathrm{Y}_{1}\left(z_{-}\right)-\mathrm{J}_{1}\left(z_{-}\right) \mathrm{Y}_{0}\left(z_{-}\right)\right] .
\end{aligned}
$$

This is a product of two Wronskians $\mathcal{W}$ (see the NIST Handbook of Mathematical functions Olver et al. (2010, formula 10.5.2)):

$$
\mathrm{J}_{0}(z) \mathrm{Y}_{1}(z)-\mathrm{J}_{1}(z) \mathrm{Y}_{0}(z)=\mathcal{W}\left\{\mathrm{J}_{0}, \mathrm{Y}_{0}\right\}=\frac{2}{\pi z} \quad \text { and because } \frac{\beta t}{\zeta^{2}+\eta^{2}}=\frac{z_{+} z_{-}}{2 r^{2}}
$$

(see definitions $((3.4 a-e)-(3.5)))$, we find the astonishing result that at all times

$$
E=T+V=\frac{1}{2}\left(\frac{1}{2 \pi r}\right)^{2}
$$

This is displayed in figure $4(d)$. Hence, the total energy is isotropic and time-independent $(\partial E / \partial t=0)$. Note this is the energy entirely contained initially in the kinetic energy of the line vortex generated by the impulsive forcing, see (3.7). At later times, kinetic energy $T$ is transferred to pseudo-potential energy $V$. This is illustrated in figure 5, which shows the angular dependence of $T / E$ and $V / E$ on a circle of radius $r=40$. Due to the self-similar development of the streamfunction field, the particular radius chosen to show its angular distribution is arbitrary. The temporal development of rapid undulations in the angular distributions of $T$ and $V$ seen in figure 5 are due to the appearance of Rossby waves of smaller and smaller scales.

How the isotropy and time-independence of $E$ come about is seen with the asymptotic properties of $T$ and $V$. They are determined by the large- $z$ behaviour of the Bessel functions in $\mathrm{I}_{m n}$ and we find that for large $\beta \operatorname{tr}$ (see appendix A):

$$
\begin{aligned}
\beta t r \rightarrow \infty: \quad T & \sim \frac{1}{2} \frac{\sin ^{2}(2 a \zeta)}{\pi^{2}\left(\zeta^{2}+\eta^{2}\right)^{2}}=\frac{1}{2} \frac{\sin ^{2}(\sqrt{2 \beta t(x+r)})}{(2 \pi r)^{2}}, \\
V & \sim \frac{1}{2} \frac{\cos ^{2}(2 a \zeta)}{\pi^{2}\left(\zeta^{2}+\eta^{2}\right)^{2}}=\frac{1}{2} \frac{\cos ^{2}(\sqrt{2 \beta t(x+r)})}{(2 \pi r)^{2}} .
\end{aligned}
$$




\section{R.C. Kloosterziel and L.R.M. Maas}

Because $\sin ^{2} z+\cos ^{2} z=1$, these approximations also yield the exact result (4.8) for the total pseudo-energy $E=T+V$. The patterns for both $T$ and $V$ have a distinct 'preference' for the westward side of the forcing - at least visually (see figures 3 and 4 ) - but when added to form $E$ this disappears and in accordance with (4.8) isotropy results. Note that this is analogous to the result for plane waves, discussed in $\S 2$, leading to constant total energy everywhere.

Kinetic energy may locally increase beyond that given by the isotropic distribution present after the instantaneous torque has been applied $(T / E>1$, see first two panels of figure 5). At the same time, pseudo-potential energy $V$ may initially become negative. This is a consequence of the material conservation of the combined planetary and relative vorticity. Advection of an initially quiescent fluid that is endowed with a meridionally varying planetary vorticity, leads to the generation of relative vorticity. Its motions enhance or diminish $T$ locally, depending on the increase or decrease of associated gradients in $G$. This is possible because the beta plane's quiescent ground state $(\boldsymbol{u}=0)$ is described from within a non-uniformly rotating frame of reference, a frame that carries non-zero ground-state energy (Tolstoy 1973). For stable wave motions, this energy may temporarily raise the kinetic energy above its initially injected amount, at the expense of $V$ turning negative. In that sense, $V$ denotes a surplus $(V>0)$ or shortage $(V<0)$ of background energy. This happens evidently without affecting that background state owing to the huge velocity difference between wave velocity and speed with which the beta plane revolves around Earth's axis. The rotating system represents an effectively infinite reservoir of energy. The presence of such a fund of energy is more easily recognized in a stable shear-flow, $U$, lacking inflection points and sheared either in the horizontal or vertical direction. Shear-flow stability waves, supported by curvature in this background flow, $U^{\prime \prime} \neq 0$, are isomorphic with Rossby waves on the beta plane (Tolstoy 1973; Harnik \& Heifetz 2007).

\section{Radial energy flux for the Green's function wave field}

In view of (2.10), when the pseudo-energy is invariant, $\partial E / \partial t=0$, the energy flux $F$ must be non-divergent:

$$
\nabla \cdot F=0
$$

everywhere outside the origin. Non-divergency of the energy flux, however, does not imply that the energy flux itself should vanish. In the presence of an initial amount of energy, introduced when the wind-stress torque is applied at $t=0$, a certain amount may escape, and this is indeed what we will find.

Using our solution for the Green's function, we can plot the energy flux vector $\boldsymbol{F}$ given in (2.10) over the plane, or, as we do here in figure 6, present it upon division by the invariant energy $E(r)$ given in (4.8). We can interpret this as the energy velocity vector $U \equiv F / E$ that can be used to construct energy streamlines and energy paths (Chapman 2001). Since the energy flux satisfies the two-dimensional continuity equation (5.1), it can be described by an energy streamfunction whose streamlines are tangent to the velocity vectors depicted in figure 6.

Figure 6 shows that energy is carried in a non-uniform manner strictly outwards, away from the source. To show this analytically, we determined the outward-directed energy flux across a circle of some fixed radius $r$ about the source-origin as a function of the azimuthal angle $\theta$. In polar coordinates, the energy flux $\boldsymbol{F}=F_{r} \boldsymbol{e}_{r}+F_{\theta} \boldsymbol{e}_{\theta}$. Here, $\boldsymbol{e}_{r}, \boldsymbol{e}_{\theta}$ denote the orthogonal unit vectors associated with the polar coordinate system. Of interest is only the radial component, $F_{r}$, which follows with the fact that $\nabla=\boldsymbol{e}_{r} \partial / \partial r+\boldsymbol{e}_{\theta}(1 / r) \partial / \partial \theta$ 
(a)

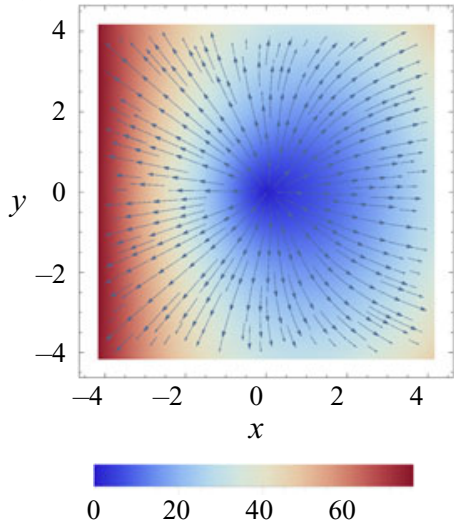

(b)

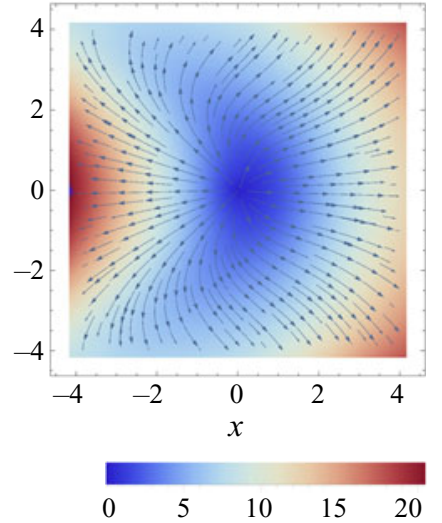

(c)

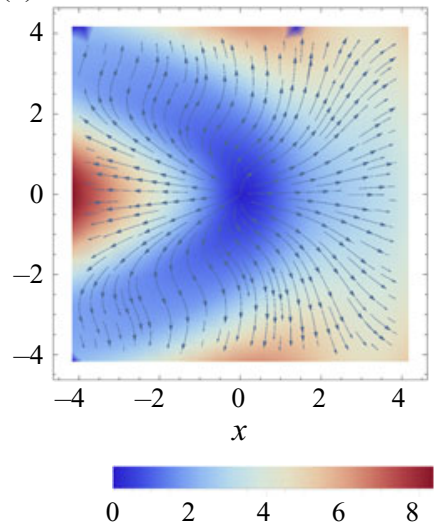

Figure 6. Energy velocity, defined as the energy flux scaled by total energy, $U=F / E$ : magnitude (colour) and direction (arrows) at times $(a) t=0.1,(b) t=0.4$ and $(c) t=1$.

Thus, with (2.10),

$$
F_{r}=-\frac{3}{2} \psi \frac{\partial^{2} \psi}{\partial t \partial r}+\frac{1}{2} \frac{\partial \psi}{\partial t} \frac{\partial \psi}{\partial r}-\beta \psi^{2} \cos \theta
$$

because $\boldsymbol{e}_{r} \cdot \boldsymbol{i}=\cos \theta$.

Since $\psi=G=\mathrm{I}_{00} / 4$ is a function of just $z_{ \pm}$(see (4.1)), the derivatives $\partial_{t}$ and $\partial_{r}$ in (5.2) are quickly calculated with the fact that according to (3.3) $\partial z_{ \pm} / \partial t=z_{ \pm} / 2 t, \partial z_{ \pm} / \partial r=$ $z_{ \pm} / 2 r$. Further, the second derivative $\partial^{2} \psi / \partial t \partial r$ can be simplified by noting that $\mathrm{J}_{0}, \mathrm{Y}_{0}$ satisfy their defining Bessel equation

$$
\left(z \frac{\partial}{\partial z}\right)^{2}\left\{\mathrm{~J}_{0}, \mathrm{Y}_{0}\right\}=-z^{2}\left\{\mathrm{~J}_{0}, \mathrm{Y}_{0}\right\}
$$

Also taking into account that $\left\{\mathrm{J}_{0}^{\prime}, \mathrm{Y}_{0}^{\prime}\right\}=-\left\{\mathrm{J}_{1}, \mathrm{Y}_{1}\right\}$ and that $z_{+}^{2}+z_{-}^{2}=2 \beta \operatorname{tr} \cos \theta$, we find

$$
F_{r}=-\frac{1}{8} \frac{1}{4^{2}} \frac{1}{t r}\left\{\left(z_{+}^{2}+z_{-}^{2}\right) \mathrm{I}_{00}^{2}+6 z_{+} z_{-} \mathrm{I}_{00} \mathrm{I}_{11}-\left[z_{+} \mathrm{I}_{10}+z_{-} \mathrm{I}_{01}\right]^{2}\right\}
$$

with the $\mathrm{I}_{m n}$ defined in (4.1). This exact result for the radial flux is shown in figure 7 as a function of $\theta$ at three instants and fixed radius $r=1$. It is seen in figure 7 that the radial component of the energy flux vector $\boldsymbol{F}$ associated with $E$ is always directed outwards, away from the source. In contrast, we find that the kinetic energy flux $\boldsymbol{F}_{T}$ for the traditional Rossby wave energy equation (2.4) exhibits inward-directed fluxes over some range of polar angles (not shown). The energy flux, $\boldsymbol{F}_{V}$, present in the conservation equation for $V$, (2.9), however, does exhibit only an outward-directed flux. This makes sense as initially all vorticity is concentrated at the source.

The complicated expression (5.4) for the radial flux can be well-approximated in the limits of small and large $\beta$ tr. Substitution in (5.4) of the small- $z$ expansions of the Bessel functions from (A1) in appendix A yields

$$
\beta t r \rightarrow 0: F_{r} \sim \frac{1}{8 \pi^{2} t r}\left[1-\frac{1}{2} \beta \operatorname{tr} \cos \theta \log ^{2}(\beta t r)+O\left((\beta t r)^{2} \log ^{2}(\beta t r)\right)\right],
$$




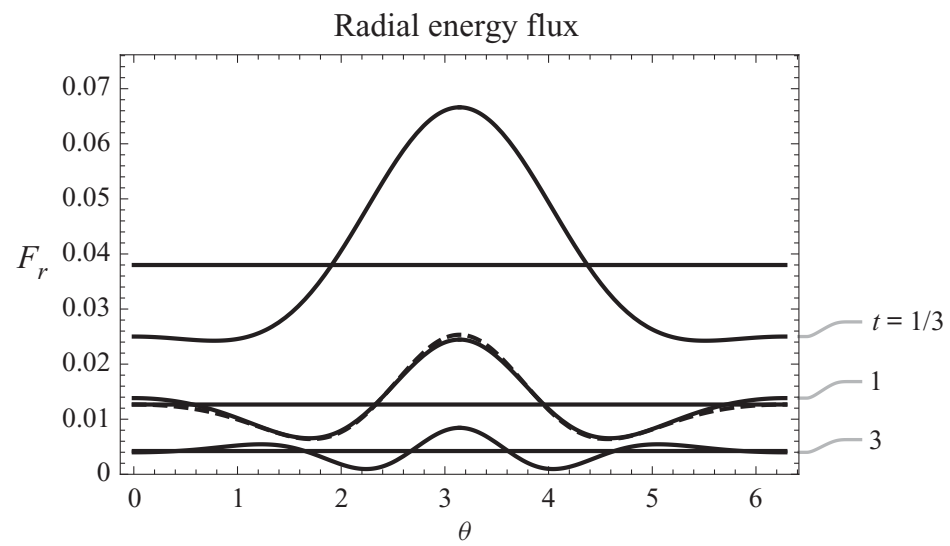

Figure 7. Radial energy flux $F_{r}$ through the unit circle $(r=1)$ at times $t=1 / 3,1,3$ as a function of azimuthal coordinate $\theta$ given by its exact expression (5.4) or (5.8) (solid) and, for $t=1$, by its asymptotic approximation (5.6) (dashed). The centre of this graph $\theta=\pi$ corresponds to west. Horizontal lines give the isotropic part $1 /\left(8 \pi^{2} t r\right)$ of $F_{r}$ at the corresponding moments.

while with substitution of the large- $z$ expansions from (A3) we find after rearrangements and use of trigonometric identities with (A5) in appendix A that

$$
\beta t r \rightarrow \infty: F_{r} \sim \frac{1}{8 \pi^{2} t r}\left[1+\sin ^{2}\left(\frac{1}{2} \theta\right) \cos \left(4 \sqrt{\beta \operatorname{tr}} \cos \left(\frac{1}{2} \theta\right)\right)\right] .
$$

This is positive for all $\theta$ and shown as dashed line in figure 7 for a single time $t=1$ at $r=1$. On the central latitude $(y=0)$

$$
\beta t r \rightarrow \infty: \text { 'east' }(\theta=0,2 \pi): F_{r} \sim \frac{1}{8 \pi^{2} t r}, \quad \text { 'west' }(\theta=\pi): F_{r} \sim \frac{1}{4 \pi^{2} t r} .
$$

In this limit along the central latitude, twice as much energy propagates to the 'west'. For small $\beta t r$, (5.5) shows that there is also a (slight) preference for westward energy propagation.

With a non-trivial analysis, we show in appendix A that when $\psi=G$ the radial flux consists of an isotropic part ( $\theta$-independent) and an anisotropic part $\mathcal{F}(r, \theta, t)$ :

$$
F_{r}=\frac{1}{8 \pi^{2} t r}+\mathcal{F}(r, \theta, t) \quad \text { with } \mathcal{F}=-\frac{2}{8} \frac{1}{4^{2}} \frac{1}{t r} \frac{\partial^{2}}{\partial \theta^{2}} \mathrm{I}_{00}^{2}=-\frac{1}{4 t r} \frac{\partial^{2}}{\partial \theta^{2}} G^{2} .
$$

The isotropic part of the outward-directed energy flux $1 /\left(8 \pi^{2} t r\right)$ is recognized in both asymptotic expressions (5.5) and (5.6).

Because of continuity of the (squared) Green's function and its azimuthal derivatives,

$$
\oint F_{r} r \mathrm{~d} \theta=\oint\left(\frac{1}{8 \pi^{2} t r}+\mathcal{F}\right) r \mathrm{~d} \theta=\oint \frac{1}{8 \pi^{2} t r} r \mathrm{~d} \theta=\frac{1}{4 \pi t}
$$

for any radius $r$. Thus, the energy flux into a domain with an inner boundary of any radius $r=r_{1}$ equals the flux out of that domain bounded by any outer, larger radius $r=r_{2}$. It agrees with the fact that $\partial E / \partial t=0$ and shows that indeed $\nabla \cdot F=0$.

Finally, let us note that the time-integrated radial energy flux as a function of direction (polar angle $\theta$ ) is indeterminate because the integral of the isotropic part, the first term in (5.8), diverges. But the anisotropic part $\int_{0}^{\tau} \mathcal{F}(r, \theta, t) \mathrm{d} t$ of the radial flux can be calculated. 


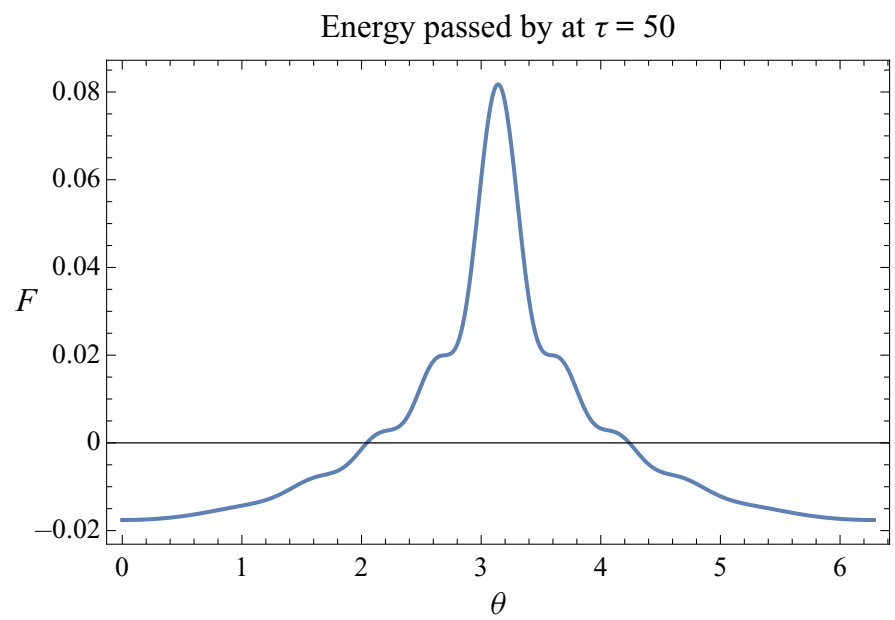

Figure 8. Azimuthal distribution of the time-integral of the anisotropic part of the radially outward-directed energy flux $F \equiv \int_{0}^{\tau} \mathcal{F}(r, \theta, t) \mathrm{d} t$ through the unit circle $r=1$. Here, $\mathcal{F}$ is defined in (5.8). The centre of this graph $\theta=\pi$ corresponds to west. The example is for $\tau=50$.

It is shown in figure 8 for time $\tau=50$. We find that as $\tau$ increases, the undulations in this curve, representing contributions of individual Rossby waves, vanish. The dominant peak in the total flux toward the western side of the forcing is obvious in figure 8 .

\section{Discussion}

The role of Rossby potential energy that Buchwald (1972) proposed nearly 50 years ago has not been firmly established. Perhaps this is because it is hard to associate the whirling motion expressed as vorticity with a form of potential energy. One might prefer to refer to it as rotational kinetic energy which it exchanges with the classical form of kinetic energy related to translation of fluid masses. Owing to its appearance in the Lagrangian density from which the Rossby wave equation can be derived by variation of its action integral (Seliger \& Whitham 1968), it seems appropriate to refer to $V$ as the pseudo-potential energy.

The present paper shows the relevance of pseudo-potential energy $V=-\left(\psi \nabla^{2} \psi\right) / 2$, both for free as well as for impulsively forced non-divergent Rossby waves. In either case, the spatio-temporal distribution of $V$ perfectly complements that of the kinetic energy distribution $T=(\nabla \psi \cdot \nabla \psi) / 2$ and leaves their sum $E=T+V$, the pseudo-energy, invariant. Remarkably, this is true at any location in the plane.

Certainly, the invariance of pseudo-energy is not a generic property of non-divergent Rossby waves, as, for example, inspection of standing waves in a rectangular basin shows. Nevertheless, the demonstrated importance of pseudo-potential energy and the appearance of a local Eulerian invariant in rotating fluids invite further investigation.

The existence of a local Eulerian isotropic invariant in a complicated spatio-temporally evolving field is a novel feature. It provides a much stronger constraint than global Eulerian invariants obtained from conservation laws do. To the best of the authors' knowledge, it is unique amongst the solutions of partial differential equations. Remarkably, the existence of this invariant is here obtained by explicit computation, in terms of products of Bessel functions. It does not seem derivable from the governing equations (2.2) and (3.1). Applying group analysis (Bluman \& Kumei 1989) to (2.2) shows that while an infinite 


\section{R.C. Kloosterziel and L.R.M. Maas}

number of conservation laws exist, none of them can be related to the local invariance of $E$ (Z. Makridin, personal communication). Further research is therefore necessary to see if this discovery of a local Eulerian invariant acts like the proverbial crack in the wall that might lead to finding local Eulerian invariants in other partial differential equations too.

From an energetic point of view, the exact Green's function (3.2) seemingly poses a riddle. How can the total pseudo-energy be both invariant, displaying merely a geometrical decay, as well as support an energy flux towards infinity? Clearly, the singularity created by the impulsive torque initially sets up a bound line vortex, characterized by a potential flow. The advection of background potential vorticity - spatially-varying planetary vorticity - leads to its evolution in the form of Rossby waves of scales and speed that are ever decreasing, folding indefinitely around the axis west of the point source. This riddle is solved by observing that subtracting an infinite amount of energy (the outward-directed energy flux) from an infinite amount of initial energy, introduced by the forcing, does not need to affect the energy distribution outside the singularity.

Our finding may throw some new light on the so-called western intensification of ocean currents occurring on a beta plane in response to variable winds. Western intensification is usually attributed to Rossby wave anisotropy and the effects that take place upon Rossby wave reflection from an ocean's western boundary. Long Rossby waves propagate their energy rapidly towards the west, while short waves propagate energy slowly to the east. When long waves reflect from an ocean's western boundary, they transfer their energy to short, eastward propagating waves having the same frequency. But these short waves practically stall while being affected by viscous and nonlinear processes. Viscosity degrades the small-scale reflected waves. Energy transported westward by long waves is therefore deposited near the ocean's western boundary. In addition, nonlinear rectification likewise intensifies when scales reduce during this reflection process, transferring wave energy into steady western boundary currents. (Pedlosky 1987).

A further clarification of the role of the beta plane on the western intensification of ocean currents is found here, not involving any boundary reflection. It might be expected that an instantaneous circularly symmetric point source sends wave energy isotropically in all directions. Here, we observe this emission to be anisotropic, favouring westward propagation of the energy flux. While confirming Stommel's conclusion that western intensification of ocean circulation must be attributed to the presence of a gradient in planetary vorticity, $\beta$, (Stommel 1948), our observation puts emphasis on the fact that anisotropy of the beta plane may well be felt right at its incipient stage, long before waves reach any ocean boundary.

An amusing corollary of this study is that the existence of a self-similar, spatio-temporally evolving Green's function that displays a local Eulerian invariant serves as a metaphor for how to reconcile two apparently contradictory pre-Socratian notions of the universe: Heraclitus' pantha rhei - everything flows / changes - versus Parmenides' permanency; despite appearances, everything exists as one, giant unchanging thing.

Acknowledgements. Critical remarks by the reviewers on the manuscript were helpful in rephrasing its content. We thank E. Heifetz for constructive comments and Z. Makridin for performing the group analysis for the free Rossby wave equation.

Funding. R.C.K. acknowledges support from National Science Foundation grant OCE 18-30098.

Declaration of interests. The authors report no conflict of interest.

Author ORCIDs.

(D) R.C. Kloosterziel https://orcid.org/0000-0002-9638-3856;

(D) L.R.M. Maas https://orcid.org/0000-0003-1523-7548.

913 A46-14 


\section{Appendix A. Mathematical details}

For small $z$,

$$
z \rightarrow 0: \quad \mathrm{J}_{0}(z) \rightarrow 1, \quad \mathrm{Y}_{0}(z) \sim(2 / \pi) \ln z, \quad \mathrm{~J}_{1} \sim \frac{z}{2}, \quad \mathrm{Y}_{1} \sim-\frac{2}{\pi z}+\frac{z}{\pi} \ln z
$$

$(\mathrm{A} 1 a-e)$

(see the NIST Handbook of Mathematical functions, Olver et al. (2010)). Substitution in (3.2) shows that therefore near the origin or at very early times $t>0$ :

$$
\operatorname{tr} \downarrow 0: G \sim \frac{1}{4}\left[\frac{2}{\pi} \ln z_{-}+\frac{2}{\pi} \ln z_{+}\right]=\frac{1}{2 \pi} \ln \beta t r=\frac{1}{2 \pi}[\ln r+\ln \beta t] .
$$

The part that is spatially constant, i.e. $(\ln \beta t) / 2 \pi$ can be dropped (but may be important in $V$ ) and therefore (3.6) follows.

For large argument $z$, it is known that for Bessel functions of order $v$

$$
z \rightarrow \infty: \mathrm{J}_{v}(z) \sim \sqrt{\frac{2}{\pi z}} \cos \left(z-\frac{1}{2} v \pi-\frac{1}{4} \pi\right), \quad \mathrm{Y}_{v}(z) \sim \sqrt{\frac{2}{\pi z}} \sin \left(z-\frac{1}{2} v \pi-\frac{1}{4} \pi\right)
$$

(see the NIST Handbook of Mathematical functions, Olver et al. (2010)). We find (3.8) after substitution of (A3) in (3.2) after use of the definitions ((3.4a-e) and (3.5)) of $z_{ \pm}$.

With the notation $\mathrm{I}_{m n}$ introduced in (4.1), with (A3),

$$
\left.\begin{array}{rl}
\beta t r \rightarrow \infty: \quad \mathrm{I}_{01}=\mathrm{J}_{0}\left(z_{+}\right) \mathrm{Y}_{1}\left(z_{-}\right)+\mathrm{J}_{1}\left(z_{-}\right) \mathrm{Y}_{0}\left(z_{+}\right) \sim-\frac{2}{\pi} \frac{\sin \left(z_{+}+z_{-}\right)}{\sqrt{z_{+} z_{-}}}, \\
\mathrm{I}_{11}=\mathrm{J}_{1}\left(z_{+}\right) \mathrm{Y}_{1}\left(z_{-}\right)+\mathrm{J}_{1}\left(z_{-}\right) \mathrm{Y}_{1}\left(z_{+}\right) \sim+\frac{2 \cos \left(z_{+}+z_{-}\right)}{\pi \sqrt{z_{+} z_{-}}},
\end{array}\right\}
$$

the asymptotics for $\mathrm{I}_{00}$ in (3.8) and noting that $\mathrm{I}_{10}$ has the same asymptotics as $\mathrm{I}_{01}$, we find in view of the definitions of $z_{ \pm}$the asymptotic expressions $((4.9 a)$ and $(4.9 b))$ for $T$ and $V$.

In the limit $\beta$ tr $\rightarrow \infty$, the radial component of the energy flux $F_{r}$ can be approximated using (3.8) and (A4) for the groups of combinations of Bessel functions $\left(\mathrm{I}_{00}, \mathrm{I}_{01}, \mathrm{I}_{10}\right.$ and $\mathrm{I}_{11}$ in (5.4)). After rearrangements of powers of $z_{ \pm}$, employing $Z \equiv z_{+}+z_{-}$, and use of the product rule for trigonometric functions, we find

$$
\begin{aligned}
F_{r} & \sim \frac{1}{8} \frac{1}{4^{2}} \frac{1}{\operatorname{tr}} \frac{1}{\pi^{2} z_{+} z_{-}}\left\{-\left(z_{+}^{2}+z_{-}^{2}\right) 4 \cos ^{2} Z-6 z_{+} z_{-}\left[-4 \cos ^{2} Z\right]+\left[\left(z_{+}+z_{-}\right)(-2 \sin Z)\right]^{2}\right\} \\
& =\frac{1}{32 t r} \frac{1}{\pi^{2} z_{+} z_{-}}\left[-\left(z_{+}-z_{-}\right)^{2} \cos \left(2\left(z_{+}+z_{-}\right)\right)+4 z_{+} z_{-}\right] .
\end{aligned}
$$

Using (3.3), in terms of cylindrical coordinates, this asymptotic expression becomes (5.6).

The expression (5.8) for $F_{r}$ was determined as follows: inspired by the way that the asymptotic expression in (A5) was formed, (5.4) can be rearranged as

$$
\begin{aligned}
F_{r} & =\frac{1}{8} \frac{1}{4^{2}} \frac{1}{t r}\left\{4 z_{+} z_{-}\left(\mathrm{I}_{10} \mathrm{I}_{01}-\mathrm{I}_{00} \mathrm{I}_{11}\right)-\left(z_{+}^{2}+z_{-}^{2}\right) \mathrm{I}_{00}^{2}-2 z_{+} z_{-} \mathrm{I}_{00} \mathrm{I}_{11}+\left[z_{+} \mathrm{I}_{10}-z_{-} \mathrm{I}_{01}\right]^{2}\right\} \\
& =\frac{1}{8} \frac{1}{4^{2}} \frac{1}{t r}\left\{\frac{4^{2}}{\pi^{2}}-\mathcal{A}(r, \theta, t)\right\},
\end{aligned}
$$


where

$$
\mathcal{A} \equiv\left(z_{+}^{2}+z_{-}^{2}\right) \mathrm{I}_{00}^{2}+2 z_{+} z_{-} \mathrm{I}_{00} \mathrm{I}_{11}-\left[z_{+} \mathrm{I}_{10}-z_{-} \mathrm{I}_{01}\right]^{2},
$$

and we used (4.4), (4.5) as well as the Wronskian expression (4.7) to simplify the first term of (A6) within the curly brackets to $4^{2} / \pi^{2}$.

With the definition of the $\mathrm{I}_{m n}$ (4.1), the following relations follow:

$$
\mathrm{I}_{10}=-\frac{\partial \mathrm{I}_{00}}{\partial z_{+}}, \quad \mathrm{I}_{01}=-\frac{\partial \mathrm{I}_{00}}{\partial z_{-}}, \quad \mathrm{I}_{11}=\frac{\partial^{2} \mathrm{I}_{00}}{\partial z_{+} \partial z_{-}}, \quad z_{ \pm}^{2} \mathrm{I}_{00}=-\left(z_{ \pm} \frac{\partial}{\partial z_{ \pm}}\right)^{2} \mathrm{I}_{00}, \quad(\mathrm{~A} 8 a-d)
$$

where we used again $\left\{\mathrm{J}_{0}^{\prime}, \mathrm{Y}_{0}^{\prime}\right\}=-\left\{\mathrm{J}_{1}, \mathrm{Y}_{1}\right\}$, and the Bessel equation (5.3). Thus, we find with the notation $L_{+}=z_{+} \partial / \partial z_{+}, L_{-}=z_{-} \partial / \partial z_{-}$

$$
\left.\begin{array}{rl}
\mathcal{A} & =-\mathrm{I}_{00}\left(L_{+}^{2}+L_{-}^{2}\right) \mathrm{I}_{00}+2 \mathrm{I}_{00}\left(L_{+} L_{-}\right) \mathrm{I}_{00}-\left[\left(L_{+}-L_{-}\right) \mathrm{I}_{00}\right]^{2} \\
& =-\mathrm{I}_{00}\left(L_{+}-L_{-}\right)^{2} \mathrm{I}_{00}-\left[\left(L_{+}-L_{-}\right) \mathrm{I}_{00}\right]^{2}=-\frac{1}{2}\left(L_{+}-L_{-}\right)^{2} \mathrm{I}_{00}^{2} \\
& =-\frac{1}{2}\left(z_{+} \frac{\partial}{\partial z_{+}}-z_{-} \frac{\partial}{\partial z_{-}}\right)^{2} \mathrm{I}_{00}^{2}=2 \frac{\partial^{2}}{\partial \theta^{2}} \mathrm{I}_{00}^{2}=2 \times 4^{2} \times \frac{\partial^{2} G}{\partial \theta^{2}}
\end{array}\right\}
$$

because, when applied to functions of the variables $\left\{z_{+}, z_{-}\right\}$,

$$
\frac{\partial}{\partial \theta}=\frac{\mathrm{i}}{2}\left(z_{+} \frac{\partial}{\partial z_{+}}-z_{-} \frac{\partial}{\partial z_{-}}\right) .
$$

Then with (A6) the result is the expression (5.8) for $F_{r}$.

\section{REFERENCES}

Bluman, G.W. \& KumeI, S. 1989 Symmetries and Differential Equations. Springer.

Buchwald, V.T. 1972 Energy and energy flux in planetary waves. Proc. R. Soc. Lond. A 328 (1572), 37-48.

ChAPMAN, C.J. 2001 Energy paths in edge waves. J. Fluid Mech. 426, 135-154.

Dickinson, R.E. 1978 Rossby waves-long-period oscillations of oceans and atmospheres. Annu. Rev. Fluid Mech. 10 (1), 159-195.

HARNIK, N. \& HEIFETZ, E. 2007 Relating overreflection and wave geometry to the counterpropagating Rossby wave perspective: toward a deeper mechanistic understanding of shear instability. J. Atmos. Sci. 64 (7), 2238-2261.

Kloosterziel, R.C. \& MaAs, L.R.M. 2017 Green's functions for Rossby waves. J. Fluid Mech. 830, 387-407.

LEBLOND, P.H. \& MYSAK, L.A. 1978 Waves in the Ocean. Elsevier.

LONGUET-Higgins, M.S. 1965 The response of a stratified ocean to stationary or moving wind-systems. Deep-Sea Res. 12, 923-973.

Olbers, D., Willebrand, J. \& Eden, C. 2012 Ocean Dynamics. Springer Science \& Business Media.ed.

Olver, F.W.J., Lozier, D.W., Boisvert, R.F. \& Clark, C.W. (Eds) 2010 NIST Handbook of Mathematical Functions. Cambridge University Press.

Pedlosky, J. 1987 Geophysical Fluid Dynamics. 2nd edn. Springer.

Seliger, R.L. \& WhithaM, G.B. 1968 Variational principles in continuum mechanics. Proc. R. Soc. Lond. A 305 (1480), 1-25.

Stommel, H. 1948 The westward intensification of wind-driven ocean currents. EOS Trans. AGU 29 (2), 202-206.

Thomson, R.E. 1973 The energy and energy flux of planetary waves in an ocean of variable depth. Geophys. Fluid Dyn. 5 (1), 385-399.

TOLSTOY, I. 1973 Wave Propagation. McGraw-Hill.

VANNESTE, J. \& SHEPHERD, T.G. 1998 On the group-velocity property for wave-activity conservation laws. J. Atmos. Sci. 55 (6), 1063-1068.

Veronis, G. 1958 On the transient response of a beta-plane ocean. J. Oceanogr. Soc. Japan 14 (1), 1-5.

Wolfram ReSEARCH, InC. 2020 Mathematica, Version 12.1. Champaign, IL, 2020. 